\title{
In Vivo Pretargeted Imaging of HER2 and TAG-72 Expression Using the HaloTag Enzyme
}

\author{
James C. Knight, ${ }^{\dagger}$ Michael Mosley, ${ }^{\dagger}$ H. Tetsuo Uyeda, ${ }^{\ddagger}$ Mei Cong, ${ }^{\ddagger}$ Frank Fan, ${ }^{\ddagger}$ Stephen Faulkner, ${ }^{\S}$ \\ and Bart Cornelissen*, ${ }^{\dagger}$ \\ ${ }^{\dagger}$ CR-UK/MRC Gray Institute for Radiation Oncology and Biology, University of Oxford, Oxford OX3 7LJ, United Kingdom \\ ${ }^{\S}$ Chemistry Research Laboratory, University of Oxford, 12 Mansfield Road, Oxford OX1 3TA, United Kingdom \\ ${ }^{\ddagger}$ Promega Corporation, Madison, Wisconsin 53711, United States
}

\section{Supporting Information}

\begin{abstract}
A novel pretargeted SPECT imaging strategy based on the HaloTag enzyme has been evaluated for the first time in a living system. To determine the efficacy of this approach, two clinically relevant cancer biomarkers, HER2 and TAG-72, were selected to represent models of internalizing and noninternalizing antigens, respectively. In MDA-MB-231/ H2N (HER2-expressing) and LS174T (TAG-72-expressing) xenograft tumors in mice, pretargeting experiments were performed in which HaloTag-conjugated derivatives of the antibodies trastuzumab (anti-HER2) or CC49 (anti-TAG-72)

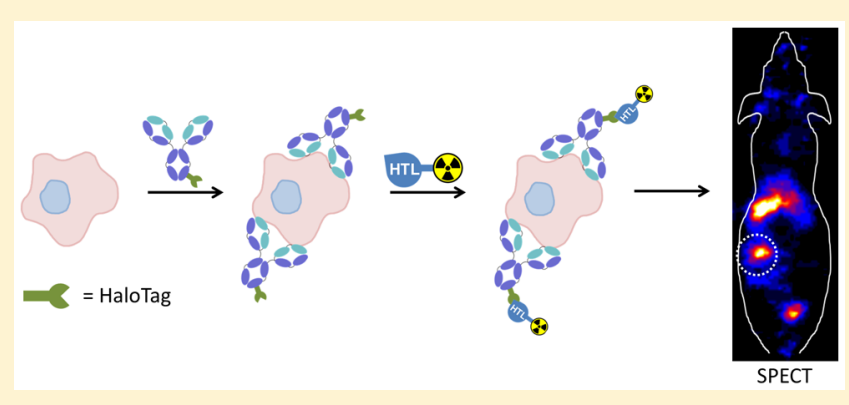
were utilized as primary agents, and the small molecule HaloTag ligands ${ }^{111}$ In-HTL-1, -2 , and -3 were evaluated as secondary agents. While this approach was not sufficiently sensitive to detect the internalizing HER2 antigen, pretargeting experiments involving the most optimal secondary agent, ${ }^{111} \mathrm{In}-\mathrm{HTL}-3$, were successful in detecting the noninternalizing antigen TAG-72 and provided high-contrast SPECT images at 4 and $24 \mathrm{~h}$ postinjection.
\end{abstract}

KEYWORDS: pretargeting, SPECT, molecular imaging, HaloTag, delivery

\section{INTRODUCTION}

Radiolabeled antibodies are frequently used in nuclear (PET/ SPECT) imaging procedures for a range of diagnostic applications. ${ }^{1}$ The long circulatory residence times of these high molecular weight $(\sim 150 \mathrm{kDa})$ constructs results in high radiation doses to patients and long waiting times before optimal target-to-blood ratios are obtained. To overcome these obstacles, there has been a considerable amount of recent effort to develop alternative two-step imaging strategies, referred to collectively as "pretargeting". In this approach, an antibody primary agent is first administered and allowed sufficient time to reach an optimal tumor-to-blood (T/B) ratio, after which a radiolabeled secondary agent in the form of a rapidly clearing small molecule is then injected. Crucially, the primary and secondary agents must be suitably designed so that they undergo extremely rapid, selective, and, preferably, irreversible binding at the tumor site. Due to the fast clearance of the radioactive secondary agent from the circulation, this leads to a lower radiation burden to the patient and $\mathrm{T} / \mathrm{B}$ contrast ratios that are comparable to or higher than those obtained by conventional radiolabeled antibodies at much earlier time points.

Pretargeting was originally achieved with bispecific antibodies that have the ability to bind to both a target antigen and a radiolabeled hapten molecule. ${ }^{3-5}$ While this approach has demonstrated some promise in clinical settings, the suboptimal binding affinities $\left(\sim 10^{-10} \mathrm{M}\right)$ that exist between the antibody and hapten species and the high production costs of bispecific antibodies have moderated the success of this approach. To improve upon this method, other pretargeting strategies have since been developed in which the primary and secondary agents are modified with complementary reactive groups that include (i) biotin/(strept)avidin, ${ }^{6-8}$ (ii) oligonucleotides (particularly those based on a morpholino backbone), ${ }^{9-11}$ and, most recently, (iii) bioorthogonal copper-free click chemistry species (particularly trans-cyclooctene/tetrazine). ${ }^{2,12}$ The most well-studied of these is the biotin/(strept)avidin pretargeting strategy; it benefits from the extremely high binding affinity interaction between the enzyme and its substrate $\left(K_{\mathrm{d}}=4 \times 10^{-14} \mathrm{M}\right)^{13,14}$ and has out-performed directly radiolabeled antibodies in clinical trials. ${ }^{15}$ However, this approach has a series of important shortcomings that impact its overall efficacy and potential for clinical translation. First, the administration of (strept)avidin-modified antibodies can potentially stimulate an adverse immunogenic response in

Received: March 6, 2017

Revised: April 28, 2017

Accepted: May 15, 2017

Published: May 15, 2017 


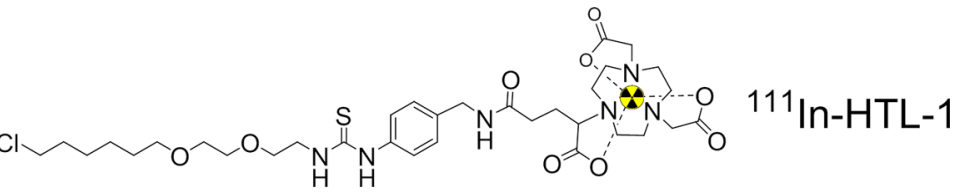

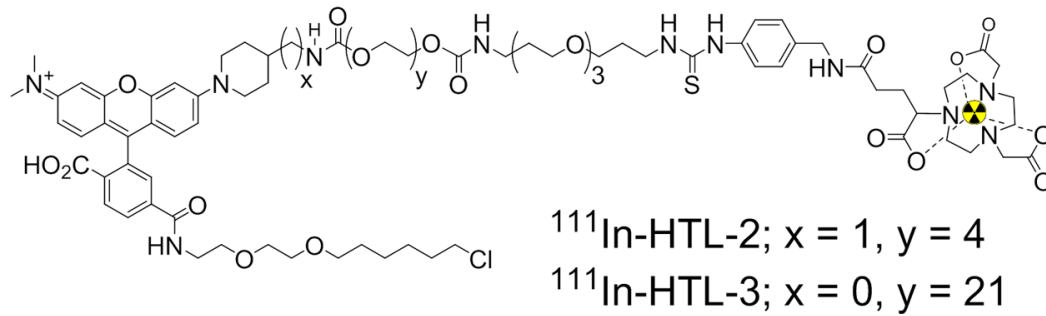

Figure 1. HaloTag ligand secondary agents used in this work ( ${ }^{111} \mathrm{In}-\mathrm{HTL}-1,-2$, and -3$)$.

patients. Second, the presence of competing endogenous biotin $\left(10^{-7}-10^{-8} \mathrm{M}\right)$ is likely to partially block or even saturate the biotin binding sites located on the primary agent, and the presence of endogenous biotinidase would also likely result in hydrolysis of the biotinylated agent. Lastly, the noncovalent nature of the interaction may result in the secondary agent gradually leaching away from the tumor site.

The reaction that occurs between the dehalogenase enzyme HaloTag $(33 \mathrm{kDa})$ and a chloroalkane HaloTag ligand (HTL; Figure 1) is well-suited to pretargeting applications. ${ }^{16,17}$ It proceeds with a second-order rate constant of $2.7 \times 10^{6} \mathrm{M}^{-1}$ $\mathrm{s}^{-1}$, which is comparable to that of the biotin/(strept)avidin reaction, ${ }^{16}$ and, importantly, it also offers a number of key advantages. For example, the HaloTag enzyme does not suffer interference from competing endogenous substrates, rendering it fully available to the HTL secondary agent. Furthermore, this enzymatic reaction also leads to the formation of a covalent bond that effectively anchors the HTL in a precise location and limits the possibility of detachment.

We and others have recently demonstrated the ability of this reaction to proceed selectively in vivo by targeting HaloTagexpressing xenograft tumors with both fluorescently labeled and radioactive imaging agents. ${ }^{18-20}$ We have also previously demonstrated in a series of in vitro experiments that HaloTag-mediated pretargeting is capable of detecting elevated expression of the cancer biomarker HER2 in a dual-modality (SPECT/optical) approach. ${ }^{19}$ Three HTLs were developed ( ${ }^{111}$ In-HTL-1, -2, and -3; Figure 1), and each was applied successfully in these in vitro pretargeting experiments. The lowest molecular weight secondary agent, ${ }^{111}$ In-HTL-1, exhibited the highest overall uptake in these experiments; however, this ligand had the highest proportion of nonspecific uptake. More promisingly, the PEGylated secondary agents, ${ }^{111}$ In-HTL-2 and -3, revealed substantially less nonspecific protein binding and higher specific binding to HER2-expressing cells. In the present study, we have used these ligands to investigate the feasibility of using the HaloTag system to enable pretargeted SPECT imaging of HER2- and TAG-72-expressing xenograft tumors in mice.

\section{MATERIALS AND METHODS}

General Methods. All reagents were purchased from Sigma-Aldrich unless otherwise stated and were used without further purification. Water was deionized using a Barnstead
NANOpure purification system (Thermo Scientific) and had a resistance of $>18.2 \mathrm{M} \Omega \mathrm{cm}^{-1}$ at $25{ }^{\circ} \mathrm{C}$. Protein concentration measurements were made on a ND-1000 spectrophotometer (NanoDrop Technologies, Inc.). $\mathrm{pH}$ was determined using $\mathrm{pH}$ indicator paper (Merck Millipore). Radioactivity measurements were made using a CRC-25R dose calibrator (Capintec, Inc.) and a 2480 WIZARD $^{2}$ or 1470 WIZARD gamma counter (PerkinElmer).

Cell Culture. The HER2-tranfected human mammary carcinoma cell line MDA-MB-231/H2N was a gift from Dr. Robert Kerbel (Sunnybrook Health Sciences Centre, Toronto, $\mathrm{ON}) .^{21}$ LS174T cells were obtained from American Type Culture Collection (ATCC). Cell lines were maintained in Dulbecco's modified Eagle's medium (DMEM, Sigma), supplemented with $10 \%$ fetal bovine serum (FBS), $2 \mathrm{mM} \mathrm{L-}$ glutamine, 100 units $/ \mathrm{mL}$ penicillin, and $0.1 \mathrm{mg} / \mathrm{mL}$ streptomycin. All cell lines were cultured in a $37{ }^{\circ} \mathrm{C}$ environment containing $5 \% \mathrm{CO}_{2}$. Cells were harvested and passaged as required using a trypsin-EDTA solution (Sigma). Cells were tested and authenticated by the providers. The cumulative length of culture was less than 6 months following retrieval from liquid nitrogen storage.

Preparation of Trastuzumab-HTP and CC49-HTP Primary Agents and HTL Secondary Agents. The primary agents in this imaging strategy were the antibody-HaloTag (Ab-HTP) conjugate proteins trastuzumab-HTP and CC49HTP. On the basis of the established antigen binding properties of the unmodified antibodies, these agents were selected to target HER2 and TAG-72 expression, respectively. Trastuzumab-HTP and CC49-HTP conjugate proteins were prepared by previously described methods using a Click\&Go proteinprotein conjugation kit (Click Chemistry Tools, catalogue no. 1008). ${ }^{19}$ The HTL secondary agents ${ }^{111}$ In-HTL-1, -2, and -3 were also synthesized and assessed for radiochemical purity via either radio-thin-layer chromatography and/or radio-HPLC following previously described methods. ${ }^{19}$ The radiochemical purity of these agents routinely exceeded $95 \%$.

In Vitro Pretargeting Studies. Aliquots of $2 \times 10^{5}$ LS174T cells were seeded in 24-well plates in warm cell culture medium $(500 \mu \mathrm{L})$. The cells were allowed to adhere overnight, and the old medium was replaced with fresh cell culture medium $(270 \mu \mathrm{L})$. Either unmodified HaloTag protein, unmodified CC49, CC49-HTP, or nonspecific rabbit IgGHTP was added to yield a final concentration of $200 \mathrm{nM}$. The 
A
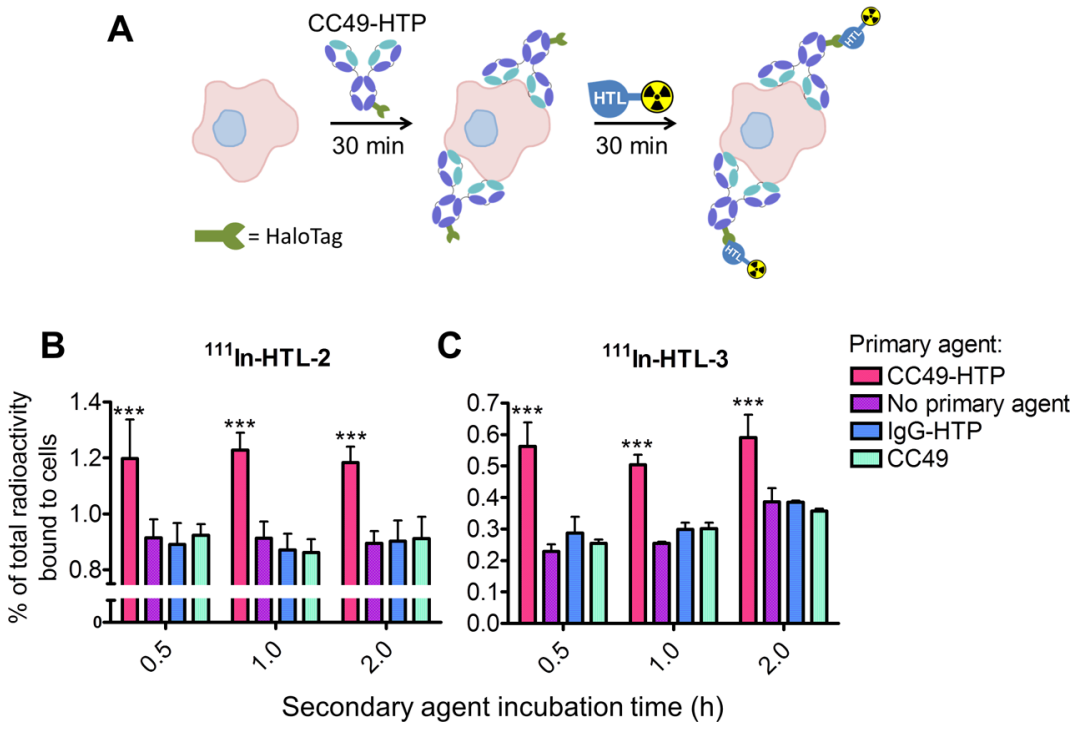

Figure 2. (A) In vitro pretargeting experiments involving (B) ${ }^{111}$ In-HTL-2 and (C) ${ }^{111}$ In-HTL-3 as secondary agents revealed an ability to detect TAG-72 expression when cells were pretargeted with CC49-HTP. ***, $P<0.001$.

cells were incubated at $37^{\circ} \mathrm{C}$ for $30 \mathrm{~min}$ at room temperature. The cell culture medium was removed, and the cells were washed twice with phosphate buffered saline (PBS; pH 7.4, 500 $\mu \mathrm{L})$. Fresh cell culture medium $(400 \mu \mathrm{L})$ was added to each well, and the relevant ${ }^{111}$ In-labeled HTL $(25 \mathrm{kBq})$ in cell culture medium $(100 \mu \mathrm{L})$ was then added. The cells were incubated for a further $30 \mathrm{~min}, 1 \mathrm{~h}$, or $2 \mathrm{~h}$ at room temperature. The cell culture medium was removed and combined with two washes of PBS ( $\mathrm{pH} 7.4,500 \mu \mathrm{L}$ ). The remaining monolayer of cells was then lysed with $0.1 \mathrm{M}$ sodium hydroxide for $20 \mathrm{~min}$ at room temperature. The amount of radioactivity contained within the cell culture medium and the cell lysate fractions was measured using a gamma counter.

In Vivo Studies. All animal procedures were performed in accordance with the UK Animals (Scientific Procedures) Act 1986 and with local ethical committee approval. Xenograft tumors were established in the right hind flank of female $\mathrm{BALB} / \mathrm{c} n u / n u$ mice by subcutaneous injection of $5 \times 10^{6}$ MDA-MB-231/H2N cells in a 1:1 mixture of fresh media and BD Matrigel basement membrane matrix (BD Biosciences) $(100 \mu \mathrm{L})$ or $5 \times 10^{6} \mathrm{LS} 174 \mathrm{~T}$ cells in sterile PBS $(100 \mu \mathrm{L})$. Subsequent SPECT/CT imaging and ex vivo biodistribution experiments were performed when the tumors had reached a volume of approximately $1 \mathrm{~cm}^{3}$.

Ex Vivo Biodistribution Experiments. TrastuzumabHTP $(100 \mu \mathrm{g})$ or CC49-HTP $(100 \mu \mathrm{g})$ in sterile PBS (100 $\mu \mathrm{L}$ ) was injected into the lateral tail vein of MDA-MB-231/ $\mathrm{H} 2 \mathrm{~N}$ or LS174T-tumor-bearing mice, respectively. Following a lag period of $24 \mathrm{~h}$, the relevant ${ }^{111}$ In-labeled HTL [ ${ }^{111}$ In-HTL-1 $(3.2 \pm 0.1 \mathrm{MBq} ; 830 \mathrm{MBq} / \mu \mathrm{mol}) ;{ }^{111} \mathrm{In}-\mathrm{HTL}-2(1.7 \pm 0.9$ $\mathrm{MBq} ; 1,170 \mathrm{MBq} / \mu \mathrm{mol}) ;{ }^{111} \mathrm{In}-\mathrm{HTL}-3(2.0 \pm 1.1 \mathrm{MBq} ; 540$ $\mathrm{MBq} / \mu \mathrm{mol})]$ was also administered via tail vein injection. Mice were then euthanized at 4 or $24 \mathrm{~h}$ postinjection (p.i.) of the HTL ligand by cervical dislocation, and selected organs, tissues, and blood were removed. The samples were immediately rinsed with water, dried, and transferred into a preweighed counting tube. After weighing, the amount of radioactivity in each tube was measured using a gamma counter. Counts per minute were converted into radioactivity units $(\mathrm{MBq})$ using calibration curves generated from known standards. These values were decay-corrected to the time of injection, and the percentage of the injected dose per gram (\% ID/g) of each sample was calculated. The xenograft tissue was then cryosectioned and analyzed by autoradiography using a Cyclone Plus phosphor imager (PerkinElmer).

SPECT Imaging Experiments. General. SPECT images were acquired using a four-head multipinhole nanoSPECT-CT scanner (Bioscan, Washington, USA) that was calibrated by imaging a phantom with an indium-111 standard solution. Images were reconstructed using InVivoScope (Bioscan), and analyses were performed using Inveon Research Workplace software package version 2.2 (Siemens Preclinical Solutions). Mice were kept under anesthesia by inhalation of $2 \%$ isofluorane in air and maintained at $37^{\circ} \mathrm{C}$.

In Vivo Imaging of TAG-72 with Directly Radiolabeled ${ }^{111}$ In-DTPA-CC49. Mice bearing LS174T xenograft tumors were administered ${ }^{111} \mathrm{In}-\mathrm{DTPA}-\mathrm{CC} 49(5 \mathrm{MBq}, 10 \mu \mathrm{g})$, and SPECT images were acquired at 24,48 , and $72 \mathrm{~h}$ p.i.

Pretargeting Experiments. At $24 \mathrm{~h}$ after injection of either trastuzumab-HTP $(100 \mu \mathrm{g})$ or CC49-HTP $(100 \mu \mathrm{g})$ into the lateral tail vein, ${ }^{111} \mathrm{In}-\mathrm{HTL}-3(3.3 \pm 1.5 \mathrm{MBq} ; 600 \mathrm{MBq} / \mu \mathrm{mol})$ was also administered via tail vein injection. SPECT images were acquired at 4 and/or $24 \mathrm{~h}$ p.i. of ${ }^{111}$ In-HTL-3.

Statistical Analyses. All statistical analyses and nonlinear regression were performed using GraphPad Prism (GraphPad Software, San Diego, CA, USA). Data were tested for normality and analyzed either by the unpaired, two-tailed Student's $t$ test where appropriate, with one- or two-way ANOVA for multiple comparisons, or with Tukey or Bonferroni post-tests, respectively, to calculate significance of differences between groups. All data were obtained at least in triplicate, and the results were reported and graphed as the mean \pm standard deviation, unless stated otherwise.

\section{RESULTS}

In Vitro Detection of TAG-72 via HaloTag-Mediated Pretargeting. Both ${ }^{111}$ In-HTL-2 and -3 enabled specific detection of TAG-72 expression on LS174T cells that had been previously incubated with CC49-HTP (Figure 2). For each HTL, maximum cell binding was achieved within an incubation 

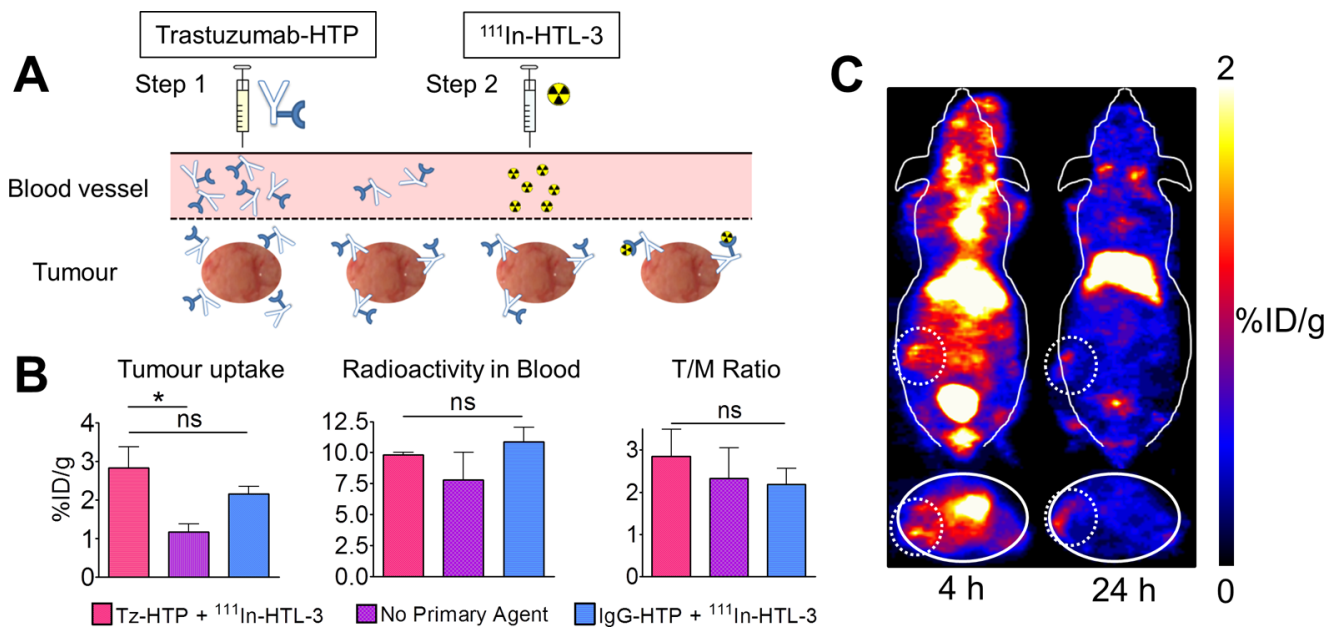

Figure 3. (A) In vivo pretargeted imaging strategy for the detection of breast cancer biomarker HER2. (B) Values obtained from ex vivo biodistribution experiments at $4 \mathrm{~h}$ p.i. of ${ }^{111}$ In-HTL-3 showing overall tumor uptake (left), radioactivity in blood (middle), and tumor-to-muscle ratios (right). ${ }^{*}, P<0.05$. (C) Representative coronal (upper) and transverse (lower) SPECT images of the same mouse obtained at 4 and $24 \mathrm{~h}$ p.i. of ${ }^{111}$ In-HTL-3. In this case, the mouse was administered trastuzumab-HTP (Tz-HTP) $24 \mathrm{~h}$ prior to the administration of ${ }^{111}$ In-HTL-3. Images intersect the center of the tumor (white dotted circle). Error bars represent the standard error of the mean.
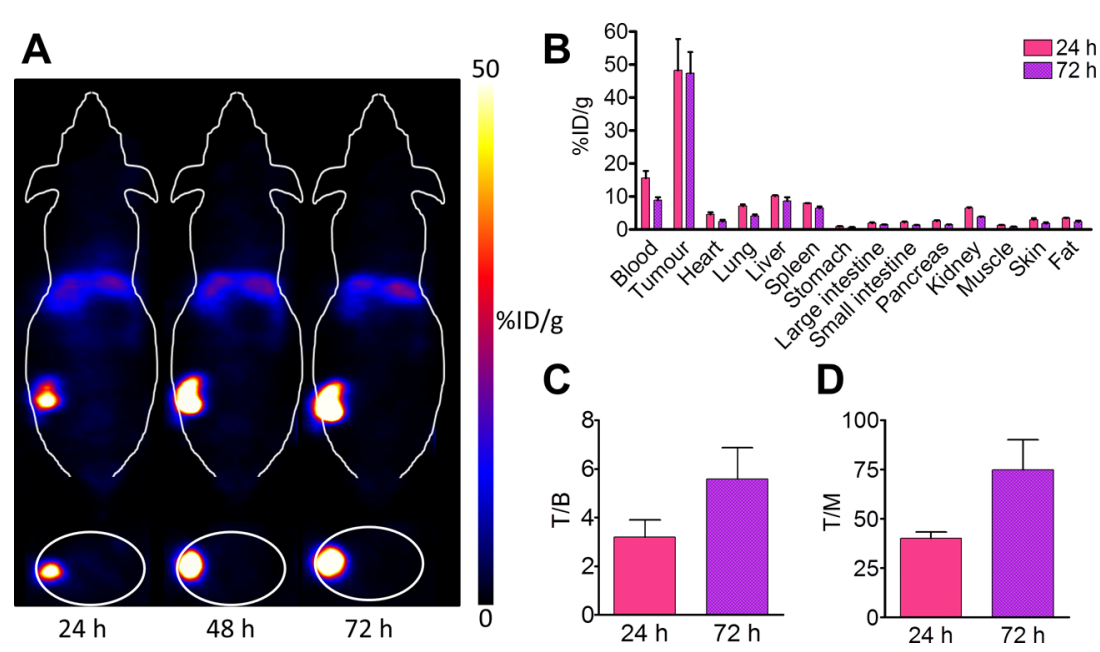

Figure 4. (A) Representative coronal (upper) and transverse (lower) SPECT images of a BALB/c nu/nu mouse bearing a TAG-72-expressing LS174T xenograft tumor (white dotted circles) acquired at 24,48 , and $72 \mathrm{~h}$ p.i. of ${ }^{111}$ In-DTPA-CC49 (5 MBq, $\left.10 \mu \mathrm{g}\right)$. (B) Values from ex vivo biodistribution experiments performed at 24 and $72 \mathrm{~h}$ p.i. (C) Tumor-to-blood ratios (T/B) and (D) tumor-to-muscle ratios (T/M) obtained from ex vivo biodistribution experiments. Error bars represent the standard error of the mean.

time of $30 \mathrm{~min}$, and no release of either HTL was observed within the $2 \mathrm{~h}$ time frame of this experiment. The total uptake of ${ }^{111} \mathrm{In}$-HTL-2 was approximately double that of ${ }^{111} \mathrm{In}-\mathrm{HTL}-3$ $(1.20 \pm 0.02$ and $0.55 \pm 0.04 \%$, respectively). Notably, however, ${ }^{111}$ In-HTL-3 exhibited considerably less nonspecific protein binding, so ${ }^{111}$ In-HTL-3 gave the highest fold increase compared with background signal (as defined by the mean average of all negative control experiments) at all time points.

In Vivo Pretargeting of HER2-Expressing Tumors. The results of ex vivo biodistribution experiments performed at $4 \mathrm{~h}$ p.i. of the HTL secondary agents revealed that uptake of ${ }^{111} \mathrm{In}$ HTL-1 and -2 was not significantly higher in the tumors of mice administered trastuzumab-HTP compared with mice given no primary agent (Tables S1 and S2). Uptake of ${ }^{111} \mathrm{In}$ HTL-3 was elevated in tumors of mice given trastuzumabHTP $(2.8 \pm 1.0 \% \mathrm{ID} / \mathrm{g})$ compared with the nonpretargeted control group $(1.2 \pm 0.4 \% \mathrm{ID} / \mathrm{g} ; P<0.05)$; however, this did not reach values significantly higher than those from mice administered a nonspecific IgG-HTP primary agent $(2.1 \pm$ $0.4 \% \mathrm{ID} / \mathrm{g}$ ) (Figure $3 \mathrm{~A}, \mathrm{~B}$ and Table S2). SPECT images acquired at $4 \mathrm{~h}$ p.i. of ${ }^{111} \mathrm{In}$-HTL-3 in mice that were previously administered trastuzumab-HTP were consistent with the biodistribution data and revealed tumors that exhibited moderate contrast against normal tissues (Figure 3C). At 24 $h$ p.i., most of the unbound ${ }^{111}$ In-HTL-3 in the blood pool was either eliminated or retained within the liver; however, uptake of ${ }^{111}$ In-HTL-3 within the tumor was still visible.

In Vivo Detection of TAG-72 Using Directly Radiolabeled ${ }^{111}$ In-DTPA-CC49. SPECT images and ex vivo biodistribution data revealed that uptake of ${ }^{111}$ In-DTPACC49 within LS174T tumors at 24 h p.i. reached $48.2 \pm 16.3 \%$ ID/g (Figure 4A,B and Table S3). At $72 \mathrm{~h}$ p.i., total uptake remained unchanged at $47.4 \pm 11.2 \% \mathrm{ID} / \mathrm{g}$; however, elimination of non-tumor-bound ${ }^{111}$ In-DTPA-CC49 led to increasing $\mathrm{T} / \mathrm{B}$ and $\mathrm{T} / \mathrm{M}$ ratios over this time frame and 


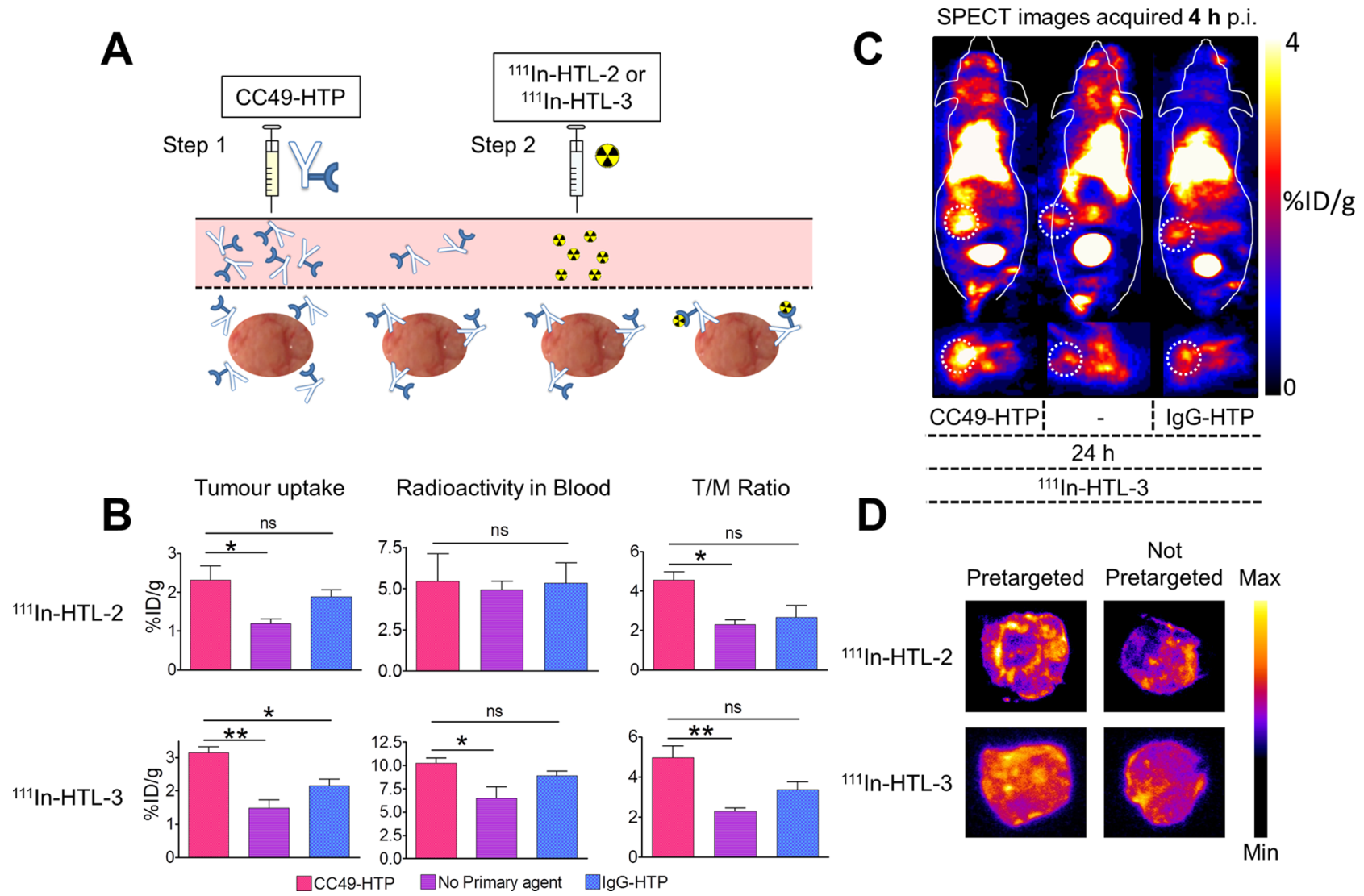

Figure 5. (A) In vivo pretargeted imaging strategy for the detection of noninternalizing antigen TAG-72. (B) Values obtained from ex vivo biodistribution experiments at $4 \mathrm{~h}$ p.i. of ${ }^{111} \mathrm{In}-\mathrm{HTL}-2$ (upper) and ${ }^{111} \mathrm{In}-\mathrm{HTL}-2$ (lower) showing overall tumor uptake (left), radioactivity in blood (middle), and tumor-to-muscle ratios (right). $*, P<0.05$; **, $P<0.01$. (C) Representative coronal (upper) and transverse (lower) SPECT images of LS174T tumor-bearing mice obtained at $4 \mathrm{~h}$ p.i. of ${ }^{111}$ In-HTL-3. In this case, the mouse was administered CC49-HTP (left), no primary agent (middle), or IgG-HTP (right) at $24 \mathrm{~h}$ prior to administration of ${ }^{111} \mathrm{In}-\mathrm{HTL}-3$. Images intersect the center of the tumor (white dotted circle). (D) Autoradiography images of harvested tumor tissue sections revealing higher uptake of ${ }^{111} \mathrm{In}$-HTL-2 and -3 in tumors of mice pretargeted with CC49-HTP. Error bars represent the standard error of the mean.

reached maximum values of $5.6 \pm 2.2$ and $74.8 \pm 26.4$, respectively (Figure 4C,D).

In Vivo Pretargeting of TAG-72-Expressing Tumors. While ${ }^{111}$ In-HTL-2 revealed higher tumor uptake in mice that received the CC49-HTP primary agent compared with that in nonpretargeted control mice at $4 \mathrm{~h}$ p.i. $(2.3 \pm 0.6$ and $1.2 \pm$ $0.2 \% \mathrm{ID} / \mathrm{g}$, respectively; $P<0.05$ ), it did not exceed the values achieved with IgG-HTP $(1.9 \pm 0.3 \% \mathrm{ID} / \mathrm{g}$ ) (Figure $5 \mathrm{~A}, \mathrm{~B}$ and Table S4). Similarly, T/M ratios followed the same trend, as values were greater in mice that received the CC49 primary agent versus no primary agent $(4.6 \pm 0.7$ and $2.3 \pm 0.4 ; P<$ $0.05)$, but values were not greater than those from mice administered IgG-HTP $(2.7 \pm 1.0)$ (Figure 5B). Tumor-toliver ratios did not differ significantly between pretargeted and nonpretargeted mice. There was also no increase in the amount of radioactivity within the blood, suggesting that any reaction product formed had already been sequestered to the tumor or cleared from the circulation within this time frame (Figure 5B). At $24 \mathrm{~h}$ p.i. of ${ }^{111} \mathrm{In}$-HTL-2, no statistical differences in tumor uptake were measured between any of the experimental groups (Table S5).

The secondary agent with the lowest nonspecific protein binding, ${ }^{111}$ In-HTL-3, reached its highest tumor uptake values in mice that received the CC49-HTP primary agent compared with mice administered either IgG-HTP or nonpretargeted control mice at 4 h p.i. $[3.2 \pm 0.3,2.1 \pm 0.3$, and $1.5 \pm 0.4 \%$
ID/g, respectively; $P<0.01$ ) (Figure 5A,B and Table S6). Tumor-to-muscle ratios were greater in mice that received the CC49 primary agent compared with no primary agent (5.0 \pm 1.0 and $2.3 \pm 0.3$, respectively; $P<0.01)$, but these ratios were not greater than those from mice administered IgG-HTP (3.4 $\pm 0.7 ; P=0.088$ ) (Figure $5 \mathrm{~B}$ ). The amount of radioactivity within the blood was higher in the CC49-HTP pretargeted group compared with the nonpretargeted group (10.2 \pm 0.9 and $6.5 \pm 2.1 \% \mathrm{ID} / \mathrm{g}$, respectively; $P<0.05$ ), which is most likely due to the formation of the enzymatic reaction product within the circulation, although this was not confirmed. As expected, the blood radioactivity levels in mice administered IgG-HTP $(8.9 \pm 0.8 \% \mathrm{ID} / \mathrm{g})$ also appeared slightly elevated compared with the nonpretargeted group, although it did not reach statistical significance. As a result of the elevated uptake of ${ }^{111} \mathrm{In}-\mathrm{HTL}-3$ in the tumors of mice administered CC49HTP, SPECT images were obtained in which tumors were clearly contrasted against surrounding tissues and organs, whereas lower tumor uptake was observed in nonpretargeted controls (Figure 5C). Autoradiography images acquired from tumor tissue sections revealed greater uptake of ${ }^{111} \mathrm{In}-\mathrm{HTL}-3$ in the tumors of mice administered the CC49-HTP primary agent versus no primary agent (Figure 5D). At 24 h p.i. of ${ }^{111} \mathrm{In}$ HTL-3, the uptake value in mice given the CC49-HTP primary agent $(3.3 \pm 0.1 \% \mathrm{ID} / \mathrm{g}$; Table S7) was comparable to that at $4 \mathrm{~h}$ p.i. and remained higher than nonspecific controls, 
yet owing to the clearance of ${ }^{111}$ In-HTL-3 from the circulation in this time frame, the tumor-to-blood contrast ratio increased markedly from 0.3 to 1.65 . The tumor-to-liver ratio was also significantly higher in mice administered CC49-HTP (0.38 \pm $0.05)$ compared with no pretargeting vector $(0.26 \pm 0.02$; $P<$ $0.05)$ and IgG-HTP $(0.28 \pm 0.05 ; P<0.05)$.

\section{DISCUSSION}

We have previously shown that pretargeting experiments involving trastuzumab-HTP and ${ }^{111}$ In-HTL-1, -2, and -3 were capable of detecting elevated expression levels of HER2 in in vitro experiments. ${ }^{19}$ In our initial efforts to translate HaloTag-mediated pretargeted imaging of HER2 into a living system, we found no evidence that the smallest secondary agents, ${ }^{111}$ In-HTL-1 and -2 , reacted with the trastuzumabHTP primary agent either within the blood or at the tumor site. This is most likely either due to the overly rapid elimination or metabolism of these agents, which in both cases would limit the possibility of reaction with HTP. More promisingly, ${ }^{111}$ In-HTL3 exhibited significantly higher binding to HER2-transfected tumors pretargeted with trastuzumab-HTP compared to nonpretargeted control experiments, although these values were not significantly higher compared with values obtained with a nonspecific IgG-HTP primary agent. Due to the high molecular weight of these primary agents, a proportion of their overall uptake in tumors (typically around $4-5 \% \mathrm{ID} / \mathrm{g}$ for a whole immunoglobulin) will be attributable to the nonspecific enhanced permeability and retention effect. As a result, modest accumulation of $\mathrm{Ab}-\mathrm{HTP}$ in tumor tissue will occur even when it is delivered with an antibody lacking antigen specificity. As a result, a general trend can be observed for all of the in vivo pretargeting experiments in which ${ }^{111}$ In-HTLs reached higher uptake values in tumors following injection of the nonspecific IgG-HTP compared with values obtained when no primary agent is administered. While the results of the HER2 pretargeting experiments indicate that a reaction between ${ }^{111}$ In-HTL-3 and the HaloTag enzyme occurred at the tumor, the comparable uptake values achieved following administration of trastuzumab-HTP and IgG-HTP suggest that this approach was not sufficiently sensitive to allow in vivo detection of HER2 expression. It should be noted that this model system is particularly challenging as it is well-established that HER2 undergoes cellular internalization upon binding trastuzumab. The rate of this internalization process $\left(t_{1 / 2}=11 \mathrm{~h}\right)^{22}$ would render approximately $80 \%$ of the primary agent inaccessible to the HTL secondary agents following a $24 \mathrm{~h}$ lag period and would therefore severely limit HER2-mediated uptake.

The efficacy of the HaloTag pretargeting approach was also examined using the noninternalizing glycoprotein TAG-72, which is overexpressed in several malignancies, including breast, ${ }^{23}$ colorectal, ${ }^{24}$ gastric, ${ }^{25}$ lung, ${ }^{26}$ ovarian, ${ }^{27}$ and pancreatic cancers. ${ }^{28,29}$ Its high cell surface persistence renders TAG-72 a more promising target for pretargeting strategies compared with HER2. ${ }^{30,31}$ The colorectal cancer cell line LS174T was selected as the basis of an in vivo model as this cell line is known to overexpress TAG-72, and when it is established as subcutaneous xenograft tumors, the expression levels of this antigen are comparable to those found in metastatic tumor masses in patients. In vitro pretargeting experiments involving the HaloTag-modified anti-TAG-72 antibody, CC49-HTP, yielded promising results as both ${ }^{111} \mathrm{In}-\mathrm{HTL}-2$ and -3 exhibited significantly higher cell binding compared with nonspecific experimental controls. While ${ }^{111}$ In-HTL-3 revealed approx- imately 50\% lower total cell binding compared with ${ }^{111}$ In-HTL2 (potentially as a result of its lower specific activity formulation), ${ }^{111}$ In-HTL-3 exhibited much lower nonspecific protein binding due to its more extensively PEGylated structure. This general trend is consistent with our previously reported in vitro pretargeting experiments involving HER2. ${ }^{19}$

In vivo pretargeting experiments in mice bearing TAG-72expressing LS174T tumors showed better efficacy compared with the previous HER2 pretargeting experiments. In this case, ${ }^{111}$ In-HTL-3 was the best performing secondary agent as at $4 \mathrm{~h}$ p.i. it accumulated to a significantly higher degree in the tumors of mice administered CC49-HTP compared with those given no primary agent or IgG-HTP. At $24 \mathrm{~h}$ after injection of ${ }^{111} \mathrm{In}$ HTL-3, no further increase in tumor uptake in mice administered CC49-HTP was observed, and these values still exceeded nonspecific experimental controls. Furthermore, as most of the non-tumor-bound ${ }^{111}$ In-HTL-3 was cleared from the blood pool, this led to a large $(450 \%)$ increase in the tumorto-blood contrast ratio and, thus, improved image quality (Figure S1). It should, however, be recognized that, compared with the directly labeled ${ }^{111}$ In-DTPA-CC49 antibody, this approach has not led to an improvement of $\mathrm{T} / \mathrm{B}$ contrast ratios. While chemical possibilities to increase the efficiency of the HaloTag reaction in vivo appear limited, additional optimization of this pretargeting approach could feasibly lead to improvements in imaging performance. In common with other pretargeting strategies, this approach is best suited to noninternalizing antigens as these provide greater accessibility to the HTL secondary agents. While we have used TAG-72 as a model system, the facile attachment of HaloTag to any antibody of interest will enable easy transposition of this method to other clinically relevant, noninternalizing cancer biomarkers, such as A33.

\section{CONCLUSIONS}

In summary, we have demonstrated for the first time that HaloTag-mediated pretargeted imaging of cancer biomarkers is possible in living animals. While this approach was not sufficiently sensitive to detect HER2 expression, it was successful in enabling detection of the noninternalizing TAG72 antigen. This novel pretargeting strategy is an important contribution to contemporary in vivo delivery systems as it represents a new addition to a very limited collection of chemical reactions that proceed in vivo. In addition to allowing targeted delivery of radioisotopes for nuclear imaging applications, the HaloTag pretargeting strategy could also be readily modified to include therapeutic radioisotopes and cytotoxic drugs.

\section{ASSOCIATED CONTENT}

\section{S Supporting Information}

The Supporting Information is available free of charge on the ACS Publications website at DOI: 10.1021/acs.molpharmaceut.7b00172.

Additional experimental methods, SPECT images, and ex vivo biodistribution data (PDF)

\section{AUTHOR INFORMATION}

\section{Corresponding Author}

*E-mail: bart.cornelissen@oncology.ox.ac.uk. Tel: +44 (0)1865 857126. Fax: +44 (0)1865 857127. 


\section{ORCID}

James C. Knight: 0000-0002-2952-5359

\section{Notes}

The authors declare the following competing financial interest(s): Some authors of this study are employees of Promega Corporation or Promega Biosciences, LLC.

\section{ACKNOWLEDGMENTS}

This research was supported by the CRUK through the Oxford Institute for Radiation Oncology and the CRUK Oxford Centre.

\section{REFERENCES}

(1) Boerman, O. C.; Oyen, W. J. G. Immuno-PET of Cancer: A Revival of Antibody Imaging. J. Nucl. Med. 2011, 52 (8), 1171-1172.

(2) van de Watering, F. C. J.; Rijpkema, M.; Robillard, M.; Oyen, W. J. G.; Boerman, O. C. Pretargeted imaging and radioimmunotherapy of cancer using antibodies and bioorthogonal chemistry. Front. Med. 2014, 1,44

(3) Goldenberg, D. M.; Rossi, E. A.; Sharkey, R. M.; McBride, W. J.; Chang, C.-H. Multifunctional Antibodies by the Dock-and-Lock Method for Improved Cancer Imaging and Therapy by Pretargeting. J. Nucl. Med. 2007, 49 (1), 158-163.

(4) Sharkey, R. M.; Karacay, H.; Goldenberg, D. M. Improving the treatment of non-Hodgkin lymphoma with antibody-targeted radionuclides. Cancer 2010, 116 (S4), 1134-1145.

(5) Goldenberg, D. M.; Chang, C.-H.; Rossi, E. A.; McBride, W. J.; Sharkey, R. M. Pretargeted Molecular Imaging and Radioimmunotherapy. Theranostics 2012, 2 (5), 523-540.

(6) Boerman, O. C.; van Schaijk, F. G.; Oyen, W. J. G.; Corstens, F. H. M. Pretargeted Radioimmunotherapy of Cancer: Progress Step by Step. J. Nucl. Med. 2003, 44 (3), 400-411.

(7) Liu, G.; Hnatowich, D. J. A Semiempirical Model of Tumor Pretargeting. Bioconjugate Chem. 2008, 19 (11), 2095-2104.

(8) Goldenberg, D. M.; Sharkey, R. M.; Paganelli, G.; Barbet, J.; Chatal, J.-F. Antibody Pretargeting Advances Cancer Radioimmunodetection and Radioimmunotherapy. J. Clin. Oncol. 2006, 24 (5), 823834.

(9) Liu, G.; Dou, S.; Liu, Y.; Wang, Y.; Rusckowski, M.; Hnatowich, D. J. 90Y labeled Phosphorodiamidate Morpholino Oligomer for Pretargeting Radiotherapy. Bioconjugate Chem. 2011, 22 (12), 25392545.

(10) Liu, G.; Dou, S.; Cheng, D.; Leif, J.; Rusckowski, M.; Streeter, P. R.; Shultz, L. D.; Hnatowich, D. J.; Greiner, D. L. Human Islet Cell MORF/cMORF Pretargeting in a Xenogeneic Murine Transplant Model. Mol. Pharmaceutics 2011, 8 (3), 767-773.

(11) He, J.; Wang, Y.; Dou, S.; Liu, X.; Zhang, S.; Liu, G.; Hnatowich, D. Affinity Enhancement Pretargeting: Synthesis and Testing of a 99mTc-Labeled Bivalent MORF. Mol. Pharmaceutics 2010, 7 (4), $1118-1124$.

(12) Knight, J. C.; Cornelissen, B. Bioorthogonal chemistry: implications for pretargeted nuclear (PET/SPECT) imaging and therapy. Am. J. Nucl. Med. Mol. Imaging 2014, 4 (2), 96-113.

(13) Hnatowich, D. J.; Virzi, F.; Rusckowski, M. Investigations of Avidin and Biotin for Imaging Applications. J. Nucl. Med. 1987, 28 (8), 1294-1302.

(14) Green, N. M. Avidin and streptavidin. Methods Enzymol. 1990, $184,51-67$

(15) Axworthy, D. B.; Fritzberg, A. R.; Hylarides, M. D.; Mallett, R. W.; Theodore, L. J.; Gustavson, L. M.; Su, F.-M.; Beaumier, P. L.; Reno, J. M. Preclinical Evaluation of An Anti-Tumor Monclonal Antibody/Streptavidin Conjugate for Pretargeted 90Y Radioimmunotherapy in A Mouse Xenograft Model. J. Immunother. 1994, 16 (2), 158.

(16) Los, G. V.; Encell, L. P.; McDougall, M. G.; Hartzell, D. D.; Karassina, N.; Zimprich, C.; Wood, M. G.; Learish, R.; Ohana, R. F.; Urh, M.; Simpson, D.; Mendez, J.; Zimmerman, K.; Otto, P.; Vidugiris,
G.; Zhu, J.; Darzins, A.; Klaubert, D. H.; Bulleit, R. F.; Wood, K. V. HaloTag: A Novel Protein Labeling Technology for Cell Imaging and Protein Analysis. ACS Chem. Biol. 2008, 3 (6), 373-382.

(17) England, C. G.; Luo, H.; Cai, W. HaloTag Technology: A Versatile Platform for Biomedical Applications. Bioconjugate Chem. 2015, 26 (6), 975-986.

(18) Tseng, J.-C.; Benink, H. A.; McDougall, M. G.; Chico-Calero, I.; Kung, A. L. In Vivo Fluorescent Labeling of Tumor Cells with the HaloTag® Technology. Curr. Chem. Genomics 2013, 6, 48-54.

(19) Knight, J. C.; Mosley, M.; Stratford, M. R. L.; Uyeda, H. T.; Benink, H. A.; Cong, M.; Fan, F.; Faulkner, S.; Cornelissen, B. Development of an enzymatic pretargeting strategy for dual-modality imaging. Chem. Commun. 2015, 51 (19), 4055-4058.

(20) Hong, H.; Benink, H. A.; Uyeda, H. T.; Valdovinos, H. F.; Zhang, Y.; Meisenheimer, P.; Barnhart, T. E.; Fan, F.; Cai, W. HaloTag as a reporter gene: positron emission tomography imaging with $64 \mathrm{Cu}-$ labeled second generation HaloTag ligands. Am. J. Transl. Res. 2013, 5 (3), 291-302.

(21) du Manoir, J. M.; Francia, G.; Man, S.; Mossoba, M.; Medin, J. A.; Viloria-Petit, A.; Hicklin, D. J.; Emmenegger, U.; Kerbel, R. S. Strategies for Delaying or Treating In vivo Acquired Resistance to Trastuzumab in Human Breast Cancer Xenografts. Clin. Cancer Res. 2006, 12 (3), 904-916.

(22) Austin, C. D.; De Mazière, A. M.; Pisacane, P. I.; van Dijk, S. M.; Eigenbrot, C.; Sliwkowski, M. X.; Klumperman, J.; Scheller, R. H. Endocytosis and Sorting of ErbB2 and the Site of Action of Cancer Therapeutics Trastuzumab and Geldanamycin. Mol. Biol. Cell 2004, 15 (12), 5268-5282.

(23) Murray, J. L.; Macey, D. J.; Grant, E. J.; Rosenblum, M. G.; Kasi, L. P.; Zhang, H.-Z.; Katz, R. L.; Rieger, P. T.; LeBherz, D.; Bhadkamkar, V.; Greiner, J. W.; Schlom, J.; Podoloff, D. A. Enhanced TAG-72 Expression and Tumor Uptake of Radiolabeled Monoclonal Antibody CC49 in Metastatic Breast Cancer Patients following $\alpha$ Interferon Treatment. Cancer Res. 1995, 55, 5925s.

(24) Xu, M.; Real, F. X.; Welt, S.; Schussler, M. H.; Oettgen, H. F.; Old, L. J. Expression of TAG-72 in normal colon, transitional mucosa, and colon cancer. Int. J. Cancer 1989, 44 (6), 985-989.

(25) Gonzalez Vitores, A. M.; Duro Ge Fau - Fraile, B. B.; Fraile Bb Fau - Carrasco, M. A.; Carrasco, M. A. Prognostic value of the glycoprotein TAG-72 in patients with gastric cancer. Int. J. Biol. Markers 2001, 16 (2), 121-125.

(26) Scott, A. M.; Wolchok, J. D.; Old, L. J. Antibody therapy of cancer. Nat. Rev. Cancer 2012, 12 (4), 278-287.

(27) Ponnusamy, M. P.; Venkatraman, G.; Singh, A. P.; Chauhan, S. C.; Johansson, S. L.; Jain, M.; Smith, L.; Davis, J. S.; Remmenga, S. W.; Batra, S. K. Expression of TAG-72 in ovarian cancer and its correlation with tumor stage and patient prognosis. Cancer Lett. 2007, 251 (2), $247-257$

(28) Takasaki, H.; Tempero, M. A.; Uchida, E.; Büchler, M.; Ness, M. J.; Burnett, D. A.; Metzgar, R. S.; Colcher, D.; Schlom, J.; Pour, P. M. Comparative studies on the expression of tumor-associated glycoprotein (TAG-72), CA 19-9 and DU-pan-2 in normal, benign and malignant pancreatic tissue. Int. J. Cancer 1988, 42 (5), 681-686.

(29) Pasquali, C.; Sperti, C.; D'Andrea, A. A.; Costantino, V.; Filipponi, C.; Pedrazzoli, S. Clinical value of seram TAG-72 as a tumor marker for pancreatic carcinoma. Int. J. Pancreatol. 1994, 15 (3), 171177.

(30) Starling, J. J.; Maciak, R. S.; Law, K. L.; Hinson, N. A.; Briggs, S. L.; Laguzza, B. C.; Johnson, D. A. In Vivo Antitumor Activity of a Monoclonal Antibody-Vinca Alkaloid Immunoconjugate Directed against a Solid Tumor Membrane Antigen Characterized by Heterogeneous Expression and Noninternalization of AntibodyAntigen Complexes. Cancer Res. 1991, 51 (11), 2965.

(31) Starling, J. J.; Law, K. L.; Hinson, N. A. Internalization Studies Using a Panel of Three Monoclonal Antibodies (MAbs) Directed Against the Human Adenocarcinoma-Associated Antigen, TAG-72. Antibody Immunoconj. Radiopharm. 1992, 5, 403-411. 